\title{
Design Space and Usability of Earable Prototyping
}

\author{
Tobias Röddiger \\ roeddiger@teco.edu \\ Karlsruhe Institute of Technology \\ Karlsruhe, Germany
}

\author{
Michael Beigl \\ beigl@teco.edu \\ Karlsruhe Institute of Technology \\ Karlsruhe, Germany
}

\author{
Anja Exler \\ exler@teco.edu \\ Karlsruhe Institute of Technology \\ Karlsruhe, Germany
}

\begin{abstract}
Earable computing gains growing attention within research and becomes ubiquitous in society. However, there is an emerging need for prototyping devices as critical drivers of innovation. In our work, we reviewed the features of existing earable platforms. Based on 24 publications, we characterized the design space of earable prototyping. We used the open eSense platform (6-axis IMU, auditory I/O) to evaluate the problem-based learning usability of non-experts. We collected data from 79 undergraduate students who developed 39 projects. Our questionnaire-based results suggest that the platform creates interest in the subject matter and supports self-directed learning. The projects align with the research space, indicating ease of use, but lack contributions for more challenging topics. Additionally, many projects included games not present in current research. The average SUS score of the platform was 67.0. The majority of problems are technical issues (e.g., connecting, playing music).
\end{abstract}

\section{CCS CONCEPTS}

\section{- Human-centered computing $\rightarrow$ Empirical studies in} ubiquitous and mobile computing.

\section{KEYWORDS}

earables; design space; problem-based learning; hearables

\section{ACM Reference Format:}

Tobias Röddiger, Michael Beigl, and Anja Exler. 2020. Design Space and Usability of Earable Prototyping. In Proceedings of the 2020 International Symposium on Wearable Computers (ISWC '20), September 12-16, 2020, Virtual Event, Mexico. ACM, New York, NY, USA, 6 pages. https://doi.org/10.1145/3410531.3414302

Permission to make digital or hard copies of all or part of this work for personal or classroom use is granted without fee provided that copies are not made or distributed for profit or commercial advantage and that copies bear this notice and the full citation on the first page. Copyrights for components of this work owned by others than the author(s) must be honored. Abstracting with credit is permitted. To copy otherwise, or republish, to post on servers or to redistribute to lists, requires prior specific permission and/or a fee. Request permissions from permissions@acm.org. ISWC '20, September 12-16, 2020, Virtual Event, Mexico

(c) 2020 Copyright held by the owner/author(s). Publication rights licensed to ACM.

ACM ISBN 978-1-4503-8077-5/20/09..\$15.00

https://doi.org/10.1145/3410531.3414302

\section{INTRODUCTION}

The ear provides exciting opportunities for human-centered applications on the head as it combines multiple advantages to realize wearables [44]. Smart ear-worn devices can quantify the user and the world around them while naturally sitting in the ear canal (e.g., $[19,38])$. Nevertheless, earables so far mostly required building custom hardware [3] or using platforms with restricted interfaces (see section 2), creating hurdles for non-experts. However, making the technology available to a broader audience might support innovation. A platform that aims to improve the availability of earable prototyping is eSense by Nokia Bell Labs [20].

We hypothesize that the eSense earbuds cover a research design space that we can structure and that it is available to non-experts in a self-learning assignment. First, our paper contributes a comparison of existing earable platforms. Based on 17 eSense publications, we propose a design space and verify it with seven articles of the other platforms. We then discuss usability when prototyping with eSense. We applied questionnaires and the system usability scale (SUS) and reviewed 39 undergraduate student projects.

\section{RELATED WORK: EARABLE PLATFORMS}

Plazak et. al. [33] reviewed the affordances of "hearables" and their possibilities for wearable computing. We focus on prototyping by inexperienced users with three requirements:

- demand no expertise in electronics or medicine

- have high-level, open data interfaces and frameworks

- require little to moderate programming experience

A platform already heavily used for problem-based education in various disciplines is Arduino [4]; however, building earables with Arduino requires substantial effort as wiring components, setting up connectivity interfaces, and fitting everything onto the ear is a challenging task already.

To summarize available earables, we initially Google Search "in-ear sensor" and "smart earbuds" and picked relevant results from the first 20 entries (earbuds with more than audio I/O). The rationale for choosing Search as opposed to, e.g., Scholar, was that market available earables are new and heavily commercialized. Table 1 presents our results. We then conducted Google Scholar based on the earable name and extract publications from the first 30 results (excluding patents / citations) that make use of any of the device capabilities. 
Table 1: Earable comparison. $(\checkmark \hat{=}$ yes, $(\checkmark) \hat{=}$ limited, $X \hat{=}$ no) (API $\hat{=}$ open app framework, FW $\hat{=}$ open firmware, MIC $\hat{=}$ microphone, SPK $\hat{=}$ speaker, IMU $\hat{=}$ inertial measurement unit, PPG $\hat{=}$ photoplythysmography, TMP $\hat{=}$ temperature sensor)

\begin{tabular}{|c|c|c|c|c|c|c|c|}
\hline Platform & API & FW & MIC & SPK & IMU & PPG & TMP \\
\hline cosinuss $^{\circ}$ & $(\sqrt{)}$ & $x$ & $x$ & $x$ & $\checkmark$ & $\checkmark$ & $\checkmark$ \\
\hline Bose & $x$ & $\checkmark$ & $\checkmark$ & $\sqrt{ }$ & $x$ & $\sqrt{ }$ & $x$ \\
\hline Soul Blade & $x$ & $x$ & $\checkmark$ & $\checkmark$ & $\checkmark$ & $\checkmark$ & $x$ \\
\hline Jabra & $x$ & $x$ & $\checkmark$ & $\checkmark$ & $\checkmark$ & $\sqrt{ }$ & $x$ \\
\hline Bragi & $(\checkmark)$ & $(\checkmark)$ & $x$ & $x$ & $x$ & $x$ & $x$ \\
\hline AirPods & $(\checkmark)$ & $x$ & $\checkmark$ & $\sqrt{ }$ & $(\checkmark)$ & $x$ & $x$ \\
\hline eSense & $\checkmark$ & $x$ & $\sqrt{ }$ & $\sqrt{ }$ & $\checkmark$ & $x$ & $x$ \\
\hline
\end{tabular}

Cosinuss $^{\circ}$ provides a highly engineered platform for medical applications with many sensors and multiple research publications [6, 10, 11, 18, 32, 37, 39]. However, data access requires proprietary software or unofficial, limited libraries. While the Bose SoundSport firmware is open, it lacks an application framework [7]. The Jabra Elite Sports have many sensors but no data access [17, 30], similar to Blade [12]. Bragi provides a powerful, closed developer framework but no more hardware [9]. Apple's AirPods have unofficial frameworks but not access to the IMU [2], similar to Google Pixel or Amazon Echo Buds. eSense [20], therefore, fills a critical gap for non-experts with a clearly defined, open framework with many research publications (see section 3). This simplification helps getting started with earables quickly.

\section{Digression: On-Boarding new Technologies}

A key motivation for making earables available to non-experts is to enable learning based on real-world projects. In that regard, the idea of problem-based learning (PBL) is to "confront students with problems from practice which provides a stimulus for learning" [8]. According to Munshi et al. [29], effective PBL should "lead to thinking, analysis, and reasoning. It should stimulate self-directed learning and fit with students' prior knowledge. The problem should show clear links with the future profession and enhance interest in the subject matter." These underlying principles will guide our evaluation.

\section{DESIGN SPACE OF EARABLE PROTOTYPING}

Smart earpieces with, e.g., an inertial measurement unit [41], photoplethysmography [43], EEG electrodes [21], etc. enable a multitude of compelling use cases. We focus on eSense, which has been available for around two years at the time of concluding this paper. Using the paper by Kawsar et al. [20] and the esense.io website, we extracted seventeen peerreviewed publications making direct use of the platform. In Figure 1, we extracted a preliminary proposal for a research design space with the primary motivation to use it for putting the non-expert projects into context. We verify that the seven publications based on other platforms presented in section 2 are also covered by Figure 1 .

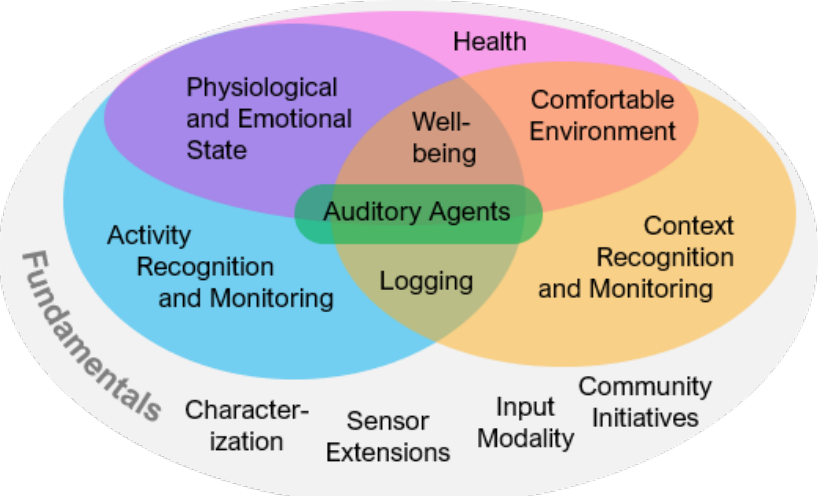

Figure 1: Initial design space characterization of publications on eSense to put into contrast with non-expert results.

In a health context, eSense was used to track the user's physiological state (respiration rate [38]). The platform was also used as mHealth building block [5] and to detect jaw clenching [40]. Activity recognition includes step counting [35], stay/walk detection as well as classifying speaking, eating and head shaking or nodding [15], drinking or chewing [23, 25], and exercising [16]. Additionally, frown and smile detection [22] as well as head movement [36] are possible indicators of the emotional state. Also, a data logging mechanism was proposed [14]. Auditory agents were used for auditive manipulation to support walking in a straight line [24]. Katayama et al. [19] proposed a setup for adapting a conversational agent's style, tone, and volume to the emotional, environmental, and social context to create a comfortable environment. Well-being creates a feedback loop, e.g., to understa conversational well-being [28]. In general, eSense can be an interface device of virtual conversational agents [1]. Work on fundamental principles creates the foundation of the research space. This includes e.g., characterization of wearing variability [27] and understanding earables as input modality, e.g., to control a robot arm [31]. Community initiatives include approaches for secure earable data sharing platforms [34], and also sensor extensions such as a magnetometer [13]. Publications for any of the other platforms also fall into the proposed prototyping design space.

\section{THE ESENSE PROTOTYPING FRAMWORK}

eSense is a stereo earable equipped with a microphone, 6axis inertial measurement unit, and dual-mode Bluetooth. It allows recording of three real-time data streams - audio, motion, and proximity (BLE RSSI). It is powered by a CSR processor, has a $40 \mathrm{mAh}$ battery, weighs 20 grams, and the dimension is $18 \times 18 \times 20 \mathrm{~mm}$, including enclosure [20,26]. Existing software libraries make it easy to get started with the earables. In our case, the students used the existing library [5] for Flutter, which is a cross-platform framework. 


\section{APPLICATION USE CASE PROJECTS}

The objective of the application use case projects was to challenge individual students with developing a mobile app that makes use of the formerly introduced eSense framework.

\section{Project Setup and Study Design}

To initially support the students with starting their projects, we decided on the following project setup:

- all students fill in an initial questionnaire reporting general information and attitude towards earables

- we introduce the task, Flutter and the eSense library

- each student gets a pair of earbuds from our lab

- students independently implement their projects

- hand in the result and fill in a second questionnaire

Otherwise, we did not limit students to any idea or particular challenge and asked them to build a creative solution that uses any of the features provided by the framework.

\section{Results}

In total, 70 students participated in building individual projects. Out of all students, 63 were male and seven female. Sixtyeight participants were undergraduate computer science and two business informatics students. 22 took the university's HCI lecture. Twenty-four students never built a mobile app before, and only five used Flutter. Twelve have used Bluetooth before. Fifty-eight students reported never having heard of earables, before we introduced them in the survey.

Suitability for Problem-based Learning. The questions we asked build upon related work on PBL [29]. Figure 2 illustrates the results of four rating scales that users filled in before the project. Overall, there is no agreement if earables are relevant for the future profession or match the overall curriculum. Key factors might be different electives and individual career plans of students. Nevertheless, the majority of students agree that the project is interesting, therefore supporting to get into the subject matter.

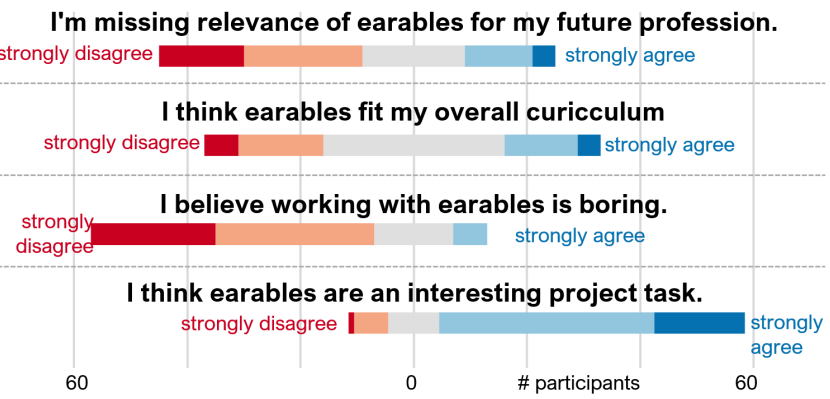

Figure 2: Rating scales filled-in by students $(n=70)$ before building projects with the eSense framework.
Retrospective Project Reflections. Unfortunately, due to COVID19 , we could only complete the retrospective questionnaire for 37 students. We report the results of the rating items in Figure 3. Overall, the project has supported self-directed learning as students strongly agree that it improved their knowledge about earables, app development, and Bluetooth Low Energy. Even though the earables did not entirely work without problems and errors occurred regularly for some, the assignment is fun. The task was not frustrating for the majority of students and Figure 5 gives further insights on problems that occured.

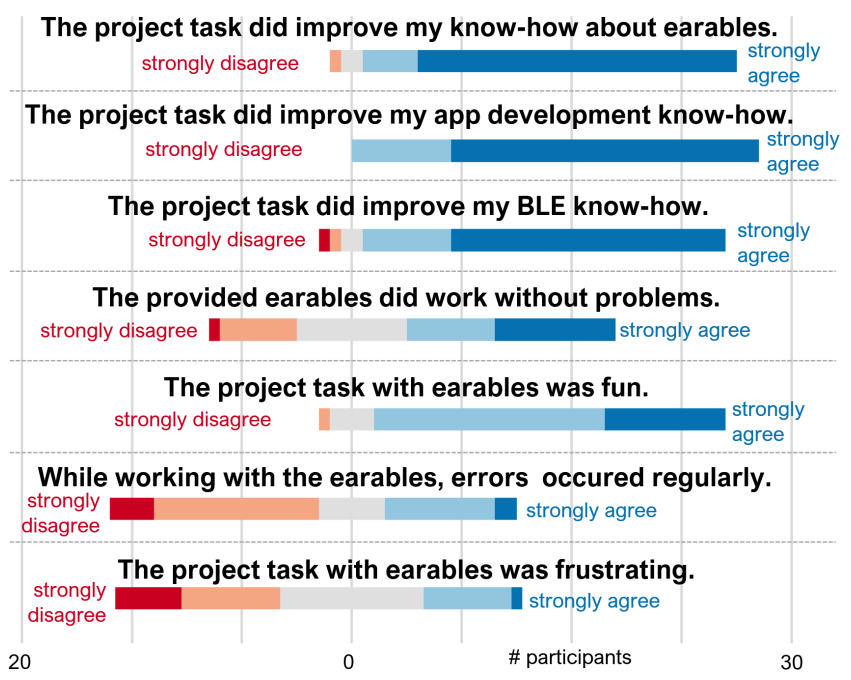

Figure 3: Results of self-directed eSense projects $(n=37)$.

Figure 4 reports the project types according to the categories defined in section 3. A topic not found in the design space analysis were games, for which earables served as direct input. Also, students built activity tracking (e.g., push-ups, situps, squats, walking, cycling, street-crossing, headbanging) with threshold state machines. One person classified nodding with ML. Auditory agents solely used textto-speech. Some students implemented physiological state tracking (e.g., sleep position and work desk body pose). The auxiliary material contains detailed project descriptions.

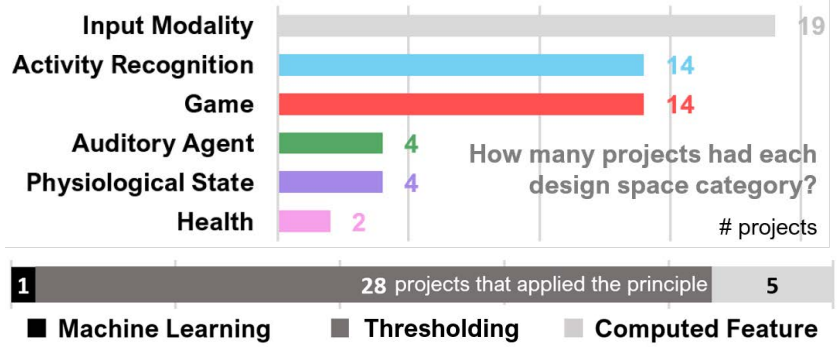

Figure 4: Project results based on category and data processing approach $(n=37)$. Projects can have multiple categories. 
Likely due to the higher complexity, no project included context recognition (e.g., use proximity, or audio features).

The overall mean SUS score was 67.0 (max: 90.0, min: 35.0, sd: 14.2), which, according to literature, is close to the average reference value of 68 [42]. For good usability, however, the score should be above 80 . In Figure 5, we present the problems that users faced extracted from free-text.

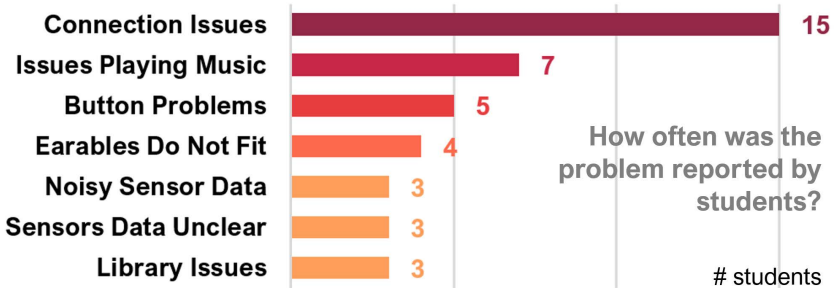

Figure 5: Problems during development extracted from free text provided by participants $(n=37)$.

Connection issues included earables taking long to connect and problems connecting both earbuds. Seven users had problems with unstable audio. Issues with the button occurred when it involuntarily triggered music, started phone calls, or sent more than one press. Especially projects with more movement reported loose fit. Only three users reported trouble understanding sensor data. Issues with the library (iOS inconsistencies) and noisy data are also very little. In contrast to the technical problems, students expressed that "the earables look modern", that it was "the best assignment they had at university so far" and also "an interesting introduction into pervasive computing”. Overall, all students successfully fulfilled the assignment. Even though the complexity of results varies, eSense was easy to use by non-experts.

\section{BLUETOOTH LIBRARY PROJECTS}

To further understand the PBL-usability of eSense, we let another group of students implement a Bluetooth library.

\section{Project Setup and Study Design}

The project and study setup was as follows:

- students gather requirements and design software

- build a Bluetooth library and sports tracking for eSense

- fill in a retrospective questionnaire about the project

\section{Results}

We recruited two teams of five students, whereas nine students voluntarily filled in the questionnaire that we sent them after project completion. They were are all undergraduate students in their second year in computer science. Both teams implemented a Bluetooth library successfully and one is available as NuGet package ${ }^{1}$ for Xamarin. Interestingly,

\footnotetext{
${ }^{1}$ https://www.nuget.org/packages/EarablesBLE/
}

for the items presented for the other project condition in Figure 3, we observe a similar trend, so we do not report them separately. However, we asked additional questions regarding the documentation of the low-level Bluetooth interfaces provided by eSense. The results are illustrated in Figure 6. Overall, the students had little trouble understanding and processing the earables' data, and also the documentation was sufficient, making eSense a good candidate for learning the fundamentals of BLE GATT.

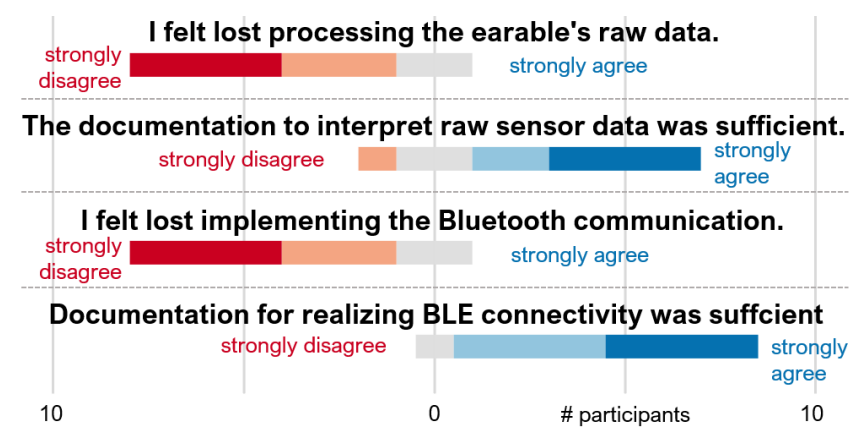

Figure 6: Rating scale results of the students implementing low-level Bluetooth libraries $(\mathbf{n}=9)$.

The SUS score for the second project setup type was not significantly different with 70 (min: 30 , max: 82.5 , sd: 16.44). The challenges that had to be overcome by the students also align with issues with connectivity and playing music.

With an allotted time frame of six months and five students per team, the final use case results closely match the shorter project assignments. Thus, the Flutter communication library significantly improves development speed.

\section{LIMITATIONS}

The initial design space that we present in our paper will likely expand in the future. Also, we did not precisely assess the time that students spent working on their solutions or if students neglected an initial idea because it was too complex.

\section{CONCLUSION AND FUTURE WORK}

Overall, earables are enablers of a broad research design space. Undergraduate CS students are excited about the subject matter and enjoy as well as succeed in building applications with the prototyping platform eSense. However, relevance for their future career is limited. Frustration mostly roots in technical issues and little problems are reported with realizing ideas. The platform's documentation for teaching in a PBL setting seems sufficient. Considering the limited technical depth of student projects, teachers should encourage responsible design (i.e., in a health context). The reference material might also include core sensor concepts, and classification libraries could simplify prototyping. 


\section{REFERENCES}

[1] Utku Günay Acer, Marc Van den Broeck, and Fahim Kawsar. 2019. The city as a personal assistant. In Adjunct Proceedings of the 2019 ACM International foint Conference on Pervasive and Ubiquitous Computing and Proceedings of the 2019 ACM International Symposium on Wearable Computers. 1102-1106.

[2] Apple. 2020. AirPods Technical Description. https://www.apple.com/ airpods-2nd-generation/specs/. Accessed: 2020-04-11.

[3] Chanavit Athavipach, Setha Pan-Ngum, and Pasin Israsena. 2019. A Wearable In-Ear EEG Device for Emotion Monitoring. Sensors 19, 18 (2019), 4014.

[4] Massimo Banzi and Michael Shiloh. 2014. Getting started with Arduino: the open source electronics prototyping platform. Maker Media, Inc.

[5] Jakob E Bardram. 2019. The CAMS eSense Framework: Enabling Earable Computing for mHealth Apps and Digital Phenotyping. In Proceedings of the 1st International Workshop on Earable Computing. 3-7.

[6] Pierre Barralon, Ivo Ramos Maia Martins, Nicole Merkle, and Stephanie Schwarz. 2015. Augmented Hearing Assistance for Elderly People. (2015).

[7] Bose. 2020. Bose SoundSport Firmware. https://github.com/ bosefirmware/ced. Accessed: 2020-04-11.

[8] David Boud and Grahame Feletti. 1998. The challenge of problem-based learning. Psychology Press.

[9] Bragi. 2020. Bragi Software. https://www.bragi.com. Accessed: 202004-11.

[10] Jorge S Chaglla E, Numan Celik, Wamadeva Balachandran, et al. 2018. Measurement of core body temperature using graphene-inked infrared thermopile sensor. Sensors 18, 10 (2018), 3315.

[11] Cosinuss. 2020. Coninuss Earables. https://www.cosinuss.com/ products/. Accessed: 2020-04-11.

[12] SOUL Electronics. 2020. BLADE True Wireless Earphones. https://www.soulelectronics.com/products/blade-sports-ai-voicecoach-true-wireless. Accessed: 2020-04-11.

[13] Andrea Ferlini, Alessandro Montanari, Cecilia Mascolo, and Robert Harle. 2019. Head Motion Tracking Through in-Ear Wearables. (2019).

[14] Alexander Hoelzemann, Henry Odoemelem, and Kristof Van Laerhoven. 2019. Using an in-ear wearable to annotate activity data across multiple inertial sensors. In Proceedings of the 1st International Workshop on Earable Computing. 14-19.

[15] Tahera Hossain, Md Shafiqul Islam, Md Atiqur Rahman Ahad, and Sozo Inoue. 2019. Human activity recognition using earable device. In Adjunct Proceedings of the 2019 ACM International foint Conference on Pervasive and Ubiquitous Computing and Proceedings of the 2019 ACM International Symposium on Wearable Computers. 81-84.

[16] Shun Ishii, Kizito Nkurikiyeyezu, Anna Yokokubo, and Guillaume Lopez. 2020. ExerSense: Real-Tme Physical Exercise Segmentation, Classification, and Counting Algorithm Using an IMU Sensor. arXiv preprint arXiv:2004.10026 (2020).

[17] Jabra. 2020. Elite Sport. https://www.jabra.com.de/sports-headphones/ jabra-elite-sport. Accessed: 2020-04-11.

[18] Denise Junger, Natividad Martínez Madrid, Nisar P Malek, and Christian Thies. 2019. Open wearables mobile platform to support personalized medicine. Telemedicine and Telemonitoring in AAL Home Environments (2019), 7.

[19] Shin Katayama, Akhil Mathur, Marc Van den Broeck, Tadashi Okoshi, Jin Nakazawa, and Fahim Kawsar. 2019. Situation-Aware Emotion Regulation of Conversational Agents with Kinetic Earables. In 2019 8th International Conference on Affective Computing and Intelligent Interaction (ACII). IEEE, 725-731.

[20] Fahim Kawsar, Chulhong Min, Akhil Mathur, and Allesandro Montanari. 2018. Earables for personal-scale behavior analytics. IEEE
Pervasive Computing 17, 3 (2018), 83-89.

[21] Preben Kidmose, David Looney, Michael Ungstrup, Mike Lind Rank, and Danilo P Mandic. 2013. A study of evoked potentials from ear-EEG. IEEE Transactions on Biomedical Engineering 60, 10 (2013), 2824-2830.

[22] Seungchul Lee, Chulhong Min, Alessandro Montanari, Akhil Mathur, Youngjae Chang, Junehwa Song, and Fahim Kawsar. 2019. Automatic Smile and Frown Recognition with Kinetic Earables. In Proceedings of the 10th Augmented Human International Conference 2019. 1-4.

[23] Roya Lotfi, George Tzanetakis, Rasit Eskicioglu, and Pourang Irani. 2020. A comparison between audio and IMU data to detect chewing events based on an earable device. In Proceedings of the 11th Augmented Human International Conference. 1-8.

[24] Kohei Matsumura and Kazushi Okada. 2019. eSense Veers: A Case Study of Acoustical Manipulation in Walking without Sight both on Subtle and Overt Conditions. In Proceedings of the 1st International Workshop on Earable Computing. 20-25.

[25] Chulhong Min, Akhil Mathur, and Fahim Kawsar. 2018. Audio-Kinetic Model for Automatic Dietary Monitoring with Earable Devices. In Proceedings of the 16th Annual International Conference on Mobile Systems, Applications, and Services. 517-517.

[26] Chulhong Min, Akhil Mathur, and Fahim Kawsar. 2018. Exploring audio and kinetic sensing on earable devices. In Proceedings of the 4th ACM Workshop on Wearable Systems and Applications. 5-10.

[27] Chulhong Min, Akhil Mathur, Alessandro Montanari, and Fahim Kawsar. 2019. An early characterisation of wearing variability on motion signals for wearables. In Proceedings of the 23rd International Symposium on Wearable Computers. 166-168.

[28] Chulhong Min, Alessandro Montanari, Akhil Mathur, Seungchul Lee, and Fahim Kawsar. 2018. Cross-modal approach for conversational well-being monitoring with multi-sensory earables. In Proceedings of the 2018 ACM International foint Conference and 2018 International Symposium on Pervasive and Ubiquitous Computing and Wearable Computers. 706-709.

[29] Fadi M Munshi, El Sayed A El Zayat, and Diana H Dolmans. 2008. Development and utility of a questionnaire to evaluate the quality of PBL problems. South East Asian fournal of Medical Education 2, 2 (2008), 32-40.

[30] Richard C Murdock and Joshua A Hagen. 2018. Soldier safety and performance through wearable devices. In Micro-and Nanotechnology Sensors, Systems, and Applications X, Vol. 10639. International Society for Optics and Photonics, 106391A.

[31] Henry Odoemelem, Alexander Hölzemann, and Kristof Van Laerhoven. 2019. Using the eSense Wearable Earbud as a Light-Weight Robot Arm Controller. (2019).

[32] Stefanie Passler, Niklas Müller, and Veit Senner. 2019. In-Ear Pulse Rate Measurement: A Valid Alternative to Heart Rate Derived from Electrocardiography? Sensors 19, 17 (2019), 3641.

[33] Joseph Plazak and Marta Kersten-Oertel. 2018. A Survey on the Affordances of "Hearables". Inventions 3, 3 (2018), 48.

[34] Jovan Powar and Alastair R Beresford. 2019. A Data Sharing Platform for Earables Research. In Proceedings of the 1st International Workshop on Earable Computing. 30-35.

[35] Jay Prakash, Zhijian Yang, Yu-Lin Wei, and Romit Roy Choudhury. 2019. STEAR: Robust Step Counting from Earables. In Proceedings of the 1st International Workshop on Earable Computing. 36-41.

[36] Sharmin Akther Purabi, Rayhan Rashed, Mirajul Islam, Nahiyan Uddin, Mahmuda Naznin, and ABM Alim Al Islam. 2019. As you are, so shall you move your head: a system-level analysis between head movements and corresponding traits and emotions. In Proceedings of the 6th International Conference on Networking, Systems and Security. 3-11. 
[37] Meera Radhakrishnan and Archan Misra. 2019. Can Earables Support Effective User Engagement during Weight-Based Gym Exercises?. In Proceedings of the 1st International Workshop on Earable Computing. 42-47.

[38] Tobias Röddiger, Daniel Wolffram, David Laubenstein, Matthias Budde, and Michael Beigl. 2019. Towards Respiration Rate Monitoring Using an In-Ear Headphone Inertial Measurement Unit. In Proceedings of the 1st International Workshop on Earable Computing. 48-53.

[39] CC Roossien, R Heus, MF Reneman, and GJ Verkerke. 2020. Monitoring core temperature of firefighters to validate a wearable non-invasive core thermometer in different types of protective clothing: concurrent in-vivo validation. Applied ergonomics 83 (2020), 103001.

[40] Siddharth Rupavatharam and Marco Gruteser. 2019. Towards In-Ear Inertial Jaw Clenching Detection. (2019).
[41] Robert S Salzar, R Cameron, and Joseph A Pellettiere. 2008. Improving earpiece accelerometer coupling to the head. SAE International fournal of Passenger Cars-Mechanical Systems 1, 2008-01-2978 (2008), 13671381.

[42] Jeff Sauro. 2011. A practical guide to the system usability scale: Background, benchmarks \& best practices. Measuring Usability LLC.

[43] Yohei Tomita and Yasue Mitsukura. 2018. An Earbud-Based Photoplethysmography and its Application. Electronics and Communications in Fapan 101, 1 (2018), 32-38.

[44] Clint Zeagler. 2017. Where to wear it: functional, technical, and social considerations in on-body location for wearable technology 20 years of designing for wearability. In Proceedings of the 2017 ACM International Symposium on Wearable Computers. 150-157. 